\title{
Electron energy-loss spectroscopy of excited states of the pyridine molecules ${ }^{\star}$
}

\author{
Ireneusz Linert ${ }^{1}$ and Mariusz Zubek ${ }^{2, a}$ \\ 1 Department of Physics of Electronic Phenomena, Faculty of Applied Physics and Mathematics, \\ Gdańsk University of Technology, 80-233 Gdańsk, Poland \\ 2 Department of Control and Power Engineering, Faculty of Ocean Engineering and Ship Technology, \\ Gdańsk University of Technology, 80-233 Gdańsk, Poland
}

Received 18 December 2015 / Received in final form 18 February 2016

Published online 5 April 2016

(C) The Author(s) 2016. This article is published with open access at Springerlink.com

\begin{abstract}
Electron energy-loss spectra of the pyridine, $\mathrm{C}_{5} \mathrm{H}_{5} \mathrm{~N}$, molecules in the gas phase have been measured to investigate electronic excitation in the energy range $3.5-10 \mathrm{eV}$. The applied wide range of residual electron energy and the scattering angle range from $10^{\circ}$ to $180^{\circ}$ enabled to differentiate between optically-allowed and -forbidden transitions. These measurements have allowed vertical excitation energies of the triplet excited states of pyridine to be determined and tentative assignments of these states to be proposed. Some of these states have not been identified in the previous works.
\end{abstract}

\section{Introduction}

The pyridine molecule, $\mathrm{C}_{5} \mathrm{H}_{5} \mathrm{~N}$, may be regarded as the simplest aromatic heterocyclic azabenzene formed by replacing one $\mathrm{CH}$ in benzene by a nitrogen atom, which means that it remains isoelectronic to benzene. The pyridine ring structure occurs in many molecules of biological importance, for example, in uracil and the nucleobases cytosine, thymine and in nicotinic acid that is related to vitamin $\mathrm{B}_{3}$ (niacin), which is found in a variety of foods. Furthermore, in the photophysics and photochemistry of hydrocarbons pyridine may serve as a benchmark molecule for investigations on the femtosecond time scale of the dynamics of the transient molecular structures of its excited electronic states $[1,2]$. The excited states of the pyridine molecule are likely to have a high degree of vibronic coupling [3]. They may deactivate via non-radiative internal conversion involving isomerization, hydrogen migration and conical intersection [4]. These extremely fast internal conversion mechanisms significantly reduce the fluorescence quantum yields from the excited states. Thus, the fluorescence of pyridine, as in the other hydrocarbons, is weak and it is difficult to record and analyze the emission spectra. Consequently, absorption measurements in pyridine provide the main source of spectroscopic data, although these principally show excitation of the opticallyallowed singlet states.

\footnotetext{
* Contribution to the Topical Issue "Advances in Positron and Electron Scattering", edited by Paulo Limao-Vieira, Gustavo Garcia, E. Krishnakumar, James Sullivan, Hajime Tanuma and Zoran Petrovic.

${ }^{a}$ e-mail: marzubek@pg.gda.pl
}

Electron impact energy-loss spectroscopy has the advantage that it is able to observe both optically-allowed and -forbidden transitions. Indeed, it allows to differentiate between the two and to facilitate the identification of the observed spectral features. This advantage of the electron energy-loss spectroscopy relies on the characteristic behavior of the excitation cross sections of the singlet and triplet states with respect to scattering angle and the incident electron energy and also on the symmetry-related selection rules [5-7]. Thus, at small scattering angles and high residual electron energy $(>10 \mathrm{eV})$ excitation of the optically-allowed states dominates the energy loss spectra. Lower residual electron energy $(<3 \mathrm{eV})$ and large scattering angles will increase the relative intensity of the optically-forbidden transitions. In particular, electron scattering at $180^{\circ}$ is expected to be governed by the electron exchange and correlation interactions and these favour singlet-triplet excitation. Moreover, spin- and symmetry-forbidden transitions usually dominate in the near-threshold spectra, which are measured at low residual electron energy $(\ll 1 \mathrm{eV})$. Furthermore, group-theoretical considerations show that in the case of symmetry-forbidden transitions, the differential excitation cross section at scattering angles of $0^{\circ}$ and $180^{\circ}$ (electron axial scattering) should be zero for diatomic molecules and relatively low for polyatomic molecules [7]. Finally, it should be pointed out, that shape and core-excited negative ion resonances may enhance the excitation of particular excited states. This is exhibited as an enhancement of the corresponding energy-loss peaks in the spectra and can aid in the monitoring of the excited states.

In the present work we have recorded electron energyloss spectra in pyridine in a wide electron energy range and 
over a range of scattering angle from $10^{\circ}$ to $180^{\circ}$. The purpose of this work is to elucidate existence of the opticallyforbidden states in pyridine and to determine their energy positions. These measurements have allowed vertical excitation energies of a number of excited states of pyridine to be determined and tentative assignments of some of these states to be proposed. Some of these states have not been identified in the previous works.

The nitrogen heteroatom integrated in the planar conjugated structure of the pyridine molecule generates the dipole moment of $2.21 \mathrm{D}$, but more importantly provides a fairly localized nitrogen lone pair orbital. These features differentiate pyridine from the benzene molecule. The pyridine molecule in the ${ }^{1} \mathrm{~A}_{1}$ ground state belongs to the $C_{2 v}$ symmetry point group and the electronic configuration for the outermost orbitals of the state may be written as $\ldots\left(6 b_{2}\right)^{2}\left(10 a_{1}\right)^{2}\left(1 b_{1}\right)^{2}\left(7 b_{2}\right)^{2}\left(2 b_{1}\right)^{2}\left(1 a_{2}\right)^{2}\left(11 a_{1}\right)^{2} \quad$ [8]. The $11 \mathrm{a}_{1}$ is the nonbonding $(n)$ nitrogen lone pair, whereas the $1 a_{2}$ and $2 b_{1}$ are the $\pi$ bonding orbitals. The two lowest unoccupied orbitals are the $3 b_{1}$ and $2 a_{2}$ and both of the these have $\pi^{*}$ character. Thus the lowest lying excited states of pyridine correspond to the $n \rightarrow \pi^{*}$ and $\pi \rightarrow \pi^{*}$ transitions. The $n \pi^{*}$ adiabatic states of the $B_{1}$ and $A_{2}$ symmetries are dominated by the $11 a_{1} \rightarrow 3 b_{1}$ and $11 a_{1} \rightarrow 2 a_{2}$ transitions, respectively. The $\pi \pi^{*}$ states have the $B_{2}$ and $A_{1}$ symmetries and involve mixtures of two $1 a_{2} \rightarrow 3 b_{1}$ and $2 b_{1} \rightarrow 2 a_{2}$ and $1 a_{2} \rightarrow 2 a_{2}$ and $2 b_{1} \rightarrow 3 b_{1}$ transitions, respectively [3]. The adiabatic energies for ionization into the first two ionic states of pyridine, $\left(11 a_{1}\right)^{-1}$ ${ }^{2} \mathrm{~A}_{1}$ and $\left(1 a_{2}\right)^{-1}{ }^{2} \mathrm{~A}_{2}$ have been very recently determined using the one-photon, mass-analyzed threshold ionization technique to be 9.1215 and $9.2750 \mathrm{eV}$, respectively [9]. The value for the $\left(1 a_{2}\right)^{-1}$ ionization energy is slightly lower than the previously measured $9.1978 \mathrm{eV}[10]$.

The ultraviolet (UV) absorption spectrum of pyridine has been studied with moderate resolution in the gas phase $[11,12]$ and in rare gas matrices [13]. The results show three distinct excitation bands of singlet states at about 5.0, 6.4 and $7.2 \mathrm{eV}$, which are assigned to ${ }^{1} \mathrm{~B}_{2}$, ${ }^{1} \mathrm{~A}_{1}$ and ${ }^{1} \mathrm{~A}_{1}+{ }^{1} \mathrm{~B}_{2}$, respectively, and some higher lying Rydberg series in the $8-9 \mathrm{eV}$ range. The data on the lower energy triplet states of pyridine were obtained from near ultraviolet singlet-triplet absorption [14], phosphorescence [15] and transient absorption [16] measurements in the gas phase and electron spin-echo investigations in benzene crystal [17]. Further data on the positions of the triplet states were provided by: electron impact threshold excitation spectra measured using the trapped-electron technique $[12,18,19]$, electron energy-loss spectra recorded (at $13 \mathrm{eV}$ incident energy) in gaseous pyridine [20] and by $\mathrm{He}^{+}$ion $(2.8 \mathrm{keV})$ energy-loss spectra in pyridine adsorbed on an $\mathrm{Ag}$ (111) surface [21]. The excitation bands related to triplet states were observed at approximately $4.1,4.84$ and $5.4 \mathrm{eV}$ and their suggested assignments are ${ }^{3} \mathrm{~A}_{1}+{ }^{3} \mathrm{~B}_{1}$, ${ }^{3} \mathrm{~A}_{1}+{ }^{3} \mathrm{~B}_{2}$ and ${ }^{3} \mathrm{~A}_{2}$, respectively. A revised and extended critical review of spectroscopic data obtained on pyridine prior to 1988 has been published by Innes et al. [22].

The singlet and triplet excited states of pyridine have been extensively studied theoretically and various

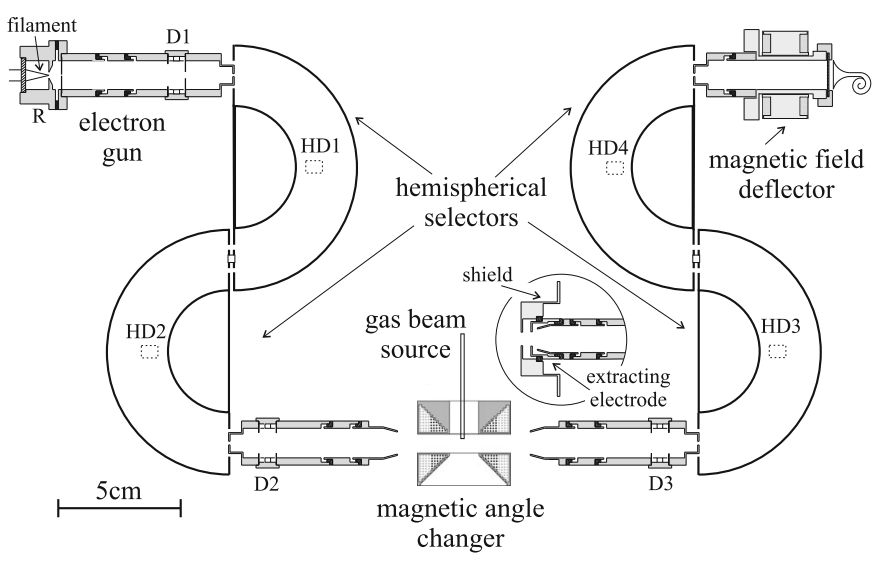

Fig. 1. Diagram of the double hemispherical electron spectrometer, which incorporates the magnetic angle changer. The entrance lens of the electron analyzer can be modified (as shown in the inset) to implement the penetrating electrostatic field technique. D1-D3 and HD1-HD4 are electron beam deflectors in the cylindrical lenses and hemispherical selectors, respectively.

advanced calculations have been performed. The most recent include: symmetry-adapted cluster configuration interaction (SAC-CI) [23-26], multiconfigurational second-order perturbation (CASPT2) [27,28], coupled cluster (CC) [3,24], time-dependent density-functional (TD-DFT) [3,25,26,29], multiconfigurational linear response (MCLR) [30] and multi-reference configuration interaction (MRCI) [12] calculations. Some of the theoretical calculations have not considered the singlet-triplet splitting of the electronic configurations [31-33]. It is generally accepted that the most advanced ab initio theoretical calculations generate results in reasonable agreement with known experimental data.

\section{Experimental methods}

\subsection{Electron spectrometer}

The electron spectrometer employed in the present measurements consists of a source of monochromatized electron beam of well defined energy, a scattered electron analyzer and a source of gas beam of the target molecules. It also incorporates a magnetic angle changer to access the full range of scattering angles from $90^{\circ}$ to $180^{\circ}$. The arrangement of the spectrometer is illustrated in Figure 1. The energy selected electrons emerging from the double-hemispherical selector of the monochromator are focused at the gas beam by a triple-electrode cylindrical lens. The scattered electron analyzer incorporates a decelerating triple-electrode cylindrical lens and a doublehemispherical selector, both of which are identical to those in the electron beam source. The analyzer can be rotated around the target beam axis over the angular range from $-90^{\circ}$ to $90^{\circ}$. A molecular beam of the pyridine is produced by a single capillary with $0.6 \mathrm{~mm}$ inner diameter by effusion of the pyridine vapour. The magnetic angle 
changer surrounds the electron scattering center and produces static, localized magnetic field whose axis of symmetry is perpendicular to the scattering plane. The magnetic field deflects the incident and the scattered electrons and enables measurements in the backward scattering from $90^{\circ}$ to $180^{\circ}$. The electron spectrometer is also equipped with an additional magnetic field deflector. This is placed between the exit of the second hemispherical selector in the scattered electrons analyzer and the entrance to the channel electron multiplier (see Fig. 1). The deflector consists of two small coils producing a magnetic field of about $10 \mathrm{G}$ that efficiently separates electrons from negative ions. It was used to determine the possible contribution of negative ions in the recorded electron spectra. Such negative ions may be formed in the dissociative attachment processes in the scattering region. The electron scattering angle $\Theta$ was calibrated against the positions of minima in the elastic differential cross sections in argon [34] with an uncertainty of $\pm 1.5^{\circ}$. The incident electron energy $E_{\mathrm{i}}$ was determined with an uncertainty of $\pm 30 \mathrm{meV}$ by observing position of the ${ }^{2} \mathrm{~S}$ negative ion resonance $(19.365 \mathrm{eV})$ in electron elastic scattering in helium. The energy resolution of the spectrometer operating in the electron energy-loss mode was in the range $40-60 \mathrm{meV}$ (FWHM). The electron energy-loss was determined to within $\pm 5 \mathrm{meV}$ using the position of the elastic scattering peak. To reduce the effect of the static magnetic field of the magnetic angle changer on the scattering angle with the increasing electron incident energy, the electron energy-loss spectra were measured in narrower energy ranges and were next matched into a single spectrum. More details on the spectrometer and its operation can be found in references $[35,36]$.

\subsection{Threshold mode of the spectrometer}

In the threshold mode of operation of the spectrometer, low energy "threshold" electrons are detected with high collection efficiency using the technique of penetrating electrostatic field [37]. The threshold electrons of low residual energy $E_{R}(<0.1 \mathrm{eV})$ are produced when the target molecules are excited just above excitation thresholds. To record threshold energy-loss spectra, the magnetic angle changer was removed and an extracting electrode together with a grounded shield was added to the deceleration cylindrical lens of the electron analyzer [38], see inset in Figure 1. The electrostatic field induced by the extracting electrode, typically held at a voltage of 80-100 V, penetrates into the collision center and produces a weak extraction field. This field collects and focuses with high efficiency low energy threshold electrons from a large solid angle, up to $4 \pi$ at energy $<$ than about $5 \mathrm{meV}$. The electron energy-loss for the threshold spectra was calibrated to within $\pm 5 \mathrm{meV}$ with respect to the $2{ }^{1} \mathrm{~S}$ excitation peak in helium, in mixture of pyridine and helium. The scattered electron residual energy $E_{R}$ was estimated from the intensity ratio of the $2{ }^{1} \mathrm{~S}$ to $2^{3} \mathrm{~S}$ helium threshold peaks [39]. The overall energy resolution of the spectrometer in these measurements was $70 \mathrm{meV}$. The background in the spectra was measured by introducing the pyridine vapour directly

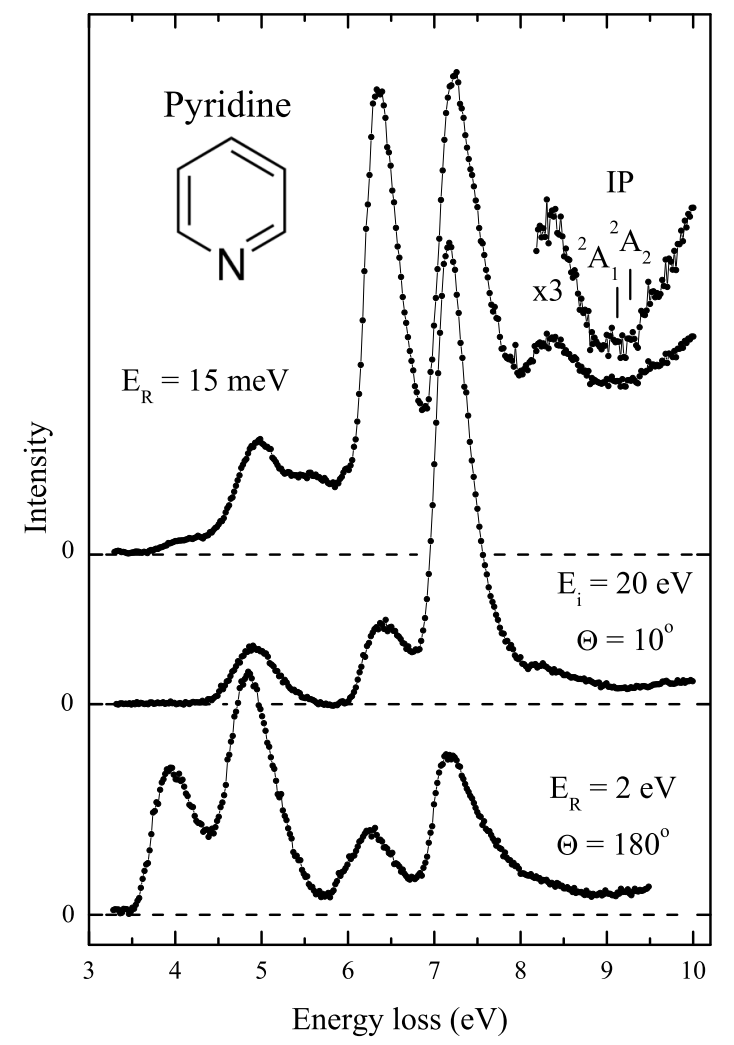

Fig. 2. Electron energy loss spectra measured in pyridine at: $E_{R}=15 \mathrm{meV}$ (threshold spectrum), $E_{i}=20 \mathrm{eV}$ and $\Theta=10^{\circ}$ and $E_{R}=2 \mathrm{eV}$ and $\Theta=180^{\circ}$.

into the vacuum chamber and bypassing the capillary. It was next subtracted from the recorded spectra.

\subsection{Pyridine beam source}

Pyridine has melting and boiling points of $-42{ }^{\circ} \mathrm{C}$ and $115^{\circ} \mathrm{C}$, respectively, and at room temperature is a liquid. The pyridine sample of stated purity of $99.8 \%$ was placed in a stainless steel vessel, which was heated to approximately $50{ }^{\circ} \mathrm{C}$ and was connected to a gas line taking the pyridine vapour into the spectrometer maintained at $60{ }^{\circ} \mathrm{C}$. The pyridine in the vessel was degassed several times under low pressure before any measurements were taken.

\section{Results}

The electron energy-loss spectra recorded in pyridine at $E_{i}=20 \mathrm{eV}$ and $\Theta=10^{\circ}, E_{R}=2 \mathrm{eV}$ and $\Theta=180^{\circ}$ and the threshold spectrum obtained at $E_{R}=15 \mathrm{meV}$ are presented in Figure 2. By using the magnetic field deflector, it was proved that the possible contribution of the negative ions into the threshold electron intensity is less than $3 \%$. The $E_{i}=20 \mathrm{eV}$ spectrum measured for forward scattering $\left(\Theta=10^{\circ}\right)$ displays three main energy-loss bands at $4.9,6.4$ and $7.2 \mathrm{eV}$ and is similar to that obtained in 


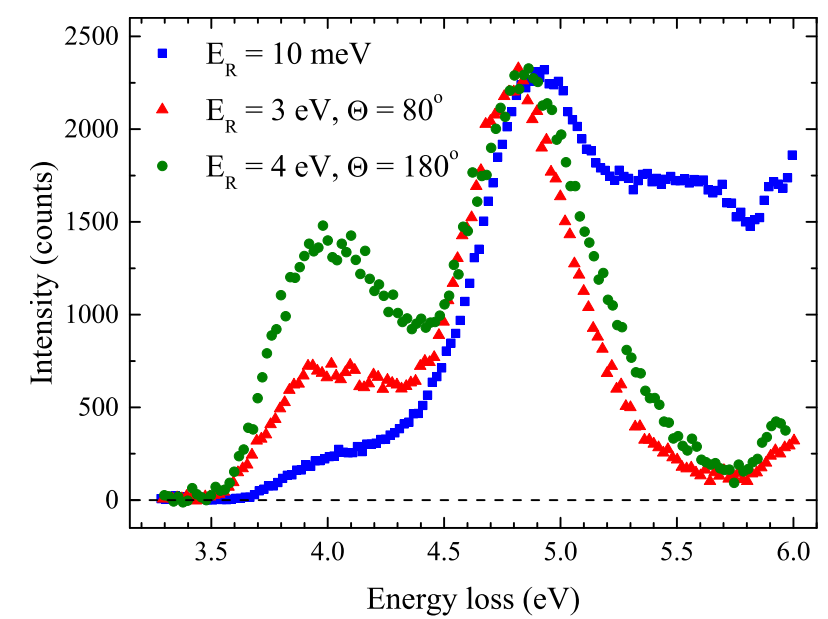

Fig. 3. Electron energy loss spectra measured in pyridine at: $E_{R}=10 \mathrm{meV}$ (threshold spectrum), $E_{R}=3 \mathrm{eV}$ and $\Theta=80^{\circ}$ and $E_{R}=4 \mathrm{eV}$ and $\Theta=180^{\circ}$.

the UV photoabsorption $[11,12]$. The spectrum, however, shows different relative intensities of the bands to those in the UV spectrum. It is in accord with energy-loss spectrum recorded earlier at $100 \mathrm{eV}$ incident energy [40]. The $E_{R}=2 \mathrm{eV}$ spectrum obtained at backward scattering $\left(\Theta=180^{\circ}\right)$ reveals the lowest lying intense energy-loss band at $4.0 \mathrm{eV}$. In this spectrum, the maxima of the 4.9 and $6.4 \mathrm{eV}$ bands are shifted now to lower energies by about 100 and $150 \mathrm{meV}$, respectively. Moreover, the origin of the $6.4 \mathrm{eV}$ peak is moved to lower energy and the $7.2 \mathrm{eV}$ band is wider and has much lower relative intensity. The $4.0 \mathrm{eV}$ energy-loss peak supports the earlier observations of excitation transitions in the $\mathrm{He}^{+}$ion $[20]$ and electron $[12,19,20]$ impacts. The $E_{R}=15 \mathrm{meV}$ threshold spectrum, in comparison to that of $E_{i}=20 \mathrm{eV}$, exhibits the maximum of the $4.9 \mathrm{eV}$ band moved by about $100 \mathrm{meV}$ to higher energy. Overlapping bands are also evident in the energy region around $5.5 \mathrm{eV}$. Further, the $6.4 \mathrm{eV}$ energyloss band significantly gained in intensity and a low intensity peak appeared at $8.3 \mathrm{eV}$. Above the first two ${ }^{2} \mathrm{~A}_{1}$ and ${ }^{2} \mathrm{~A}_{2}$ ionization thresholds of pyridine, the electron intensity shows an increase. The above and other energy-loss spectra measured in this work exhibit evidence that strong variations of the shapes of the spectra with the scattering conditions are apparent. The variations of the spectra in the region up to $6 \mathrm{eV}$ are displayed in detail in Figure 3, which clearly suggests that a number of pyridine electronic states, including the triplet states, are excited.

The measured energy-loss spectra were analyzed using a least-square fitting routine and applying a number of Gaussian profiles to discern the excitation bands in the energy range $3.5-8 \mathrm{eV}$ corresponding to valence and Rydberg electronic states of pyridine. However, the UV spectra $[11,12]$ and the present energy-loss spectra measured at conditions, which favour excitation of the optically-allowed transitions, indicate that the 4.9, 6.4 and $7.2 \mathrm{eV}$ excitation bands display asymmetric shapes. These bands were approximated by the bi-Gaussian profiles that is functions describing each wing of the profile by its own width. The initial series of the profiles, that was introduced into the fitting was constructed from the bands of the singlet states derived from the UV photoabsorption measurements and the new bands in the energy-loss spectra, which may correspond to the optically-forbidden transitions. For example, the $4.9 \mathrm{eV}$ band was approximated by two profiles, a Gaussian and a bi-Gaussian profiles, following the observation of two singlet states in the UV spectrum [11]. The $4.0 \mathrm{eV}$ energy-loss band, on the ground of its variation with $E_{R}$ and $\Theta$, was approximated by two Gaussians. The constructed set of twelve initial profiles describing the energy-loss spectra were optimized to obtain the best fit to the all fourteen measured spectra. This was achieved by carrying out single fits in narrower, but overlapping, energy ranges $(\sim 1.5 \mathrm{eV}$ wide) in the individual spectra. These steps reduced the number of simultaneously determined parameters, energies and widths, of the functions and increased reliability of the fitting. The single fits were reiterated over the whole energy regions of the spectra and the fitting routine was interrupted when a satisfactory agreement was obtained for all experimental energy-loss spectra. It is worth noting that the energy positions and widths of the excitation bands deduced from the fourteen individual energy-loss spectra deviated from their average values by not more than \pm 30 and $\pm 50 \mathrm{meV}$, respectively. The same procedure was applied in our recent study of electronic excitation of pyrimidine [41]. Figures 4 and 5 present the quality of fitting and the positions of the inferred excitation bands in the energy regions below $6 \mathrm{eV}$ and above $5.5 \mathrm{eV}$, respectively, in the selected spectra.

The derived vertical excitation energies of the electronic states of pyridine are listed in Table 1. Their maximum uncertainty is $\pm 30 \mathrm{meV}$. These energies are compared with those obtained in the UV absorption, electron impact measurements and in the recent calculations. Table 1 also contains assignments of the singlet states following reference [22] and suggested tentative assignments of the observed states of the optically-forbidden transitions.

\section{Discussion}

The first two singlet states, assigned to $n \pi{ }^{*}{ }^{1} \mathrm{~B}_{1}$ and $\pi \pi^{*}$ ${ }^{1} \mathrm{~B}_{2}$ symmetries [11,22], are observed in the present spectra at 4.78 and $4.99 \mathrm{eV}$ (vertical excitation energies), respectively. The excitation of these two close-lying states yields a single $4.9 \mathrm{eV}$ band in the energy-loss spectra. This band recorded at conditions which favour opticallyallowed transitions has a nearly identical shape to that obtained in absorption by Bolovinos et al. [11], although it is shifted to lower energy (see Fig. 4a). The varying contribution of the ${ }^{1} \mathrm{~B}_{1}$ state to the $4.9 \mathrm{eV}$ band results in the maximum of the band shifting in energy by about $\pm 100 \mathrm{meV}$ in the spectra measured at different electron residual energies, as illustrated in Figure 3. The present ${ }^{1} \mathrm{~B}_{1}$ vertical excitation energy of $4.78 \mathrm{eV}$ has been obtained from the first clear identification of the ${ }^{1} \mathrm{~B}_{1}$ band. This result supports the previously estimated energy of $4.74 \mathrm{eV}$ by Cai and Reimers [3]. These authors obtained 


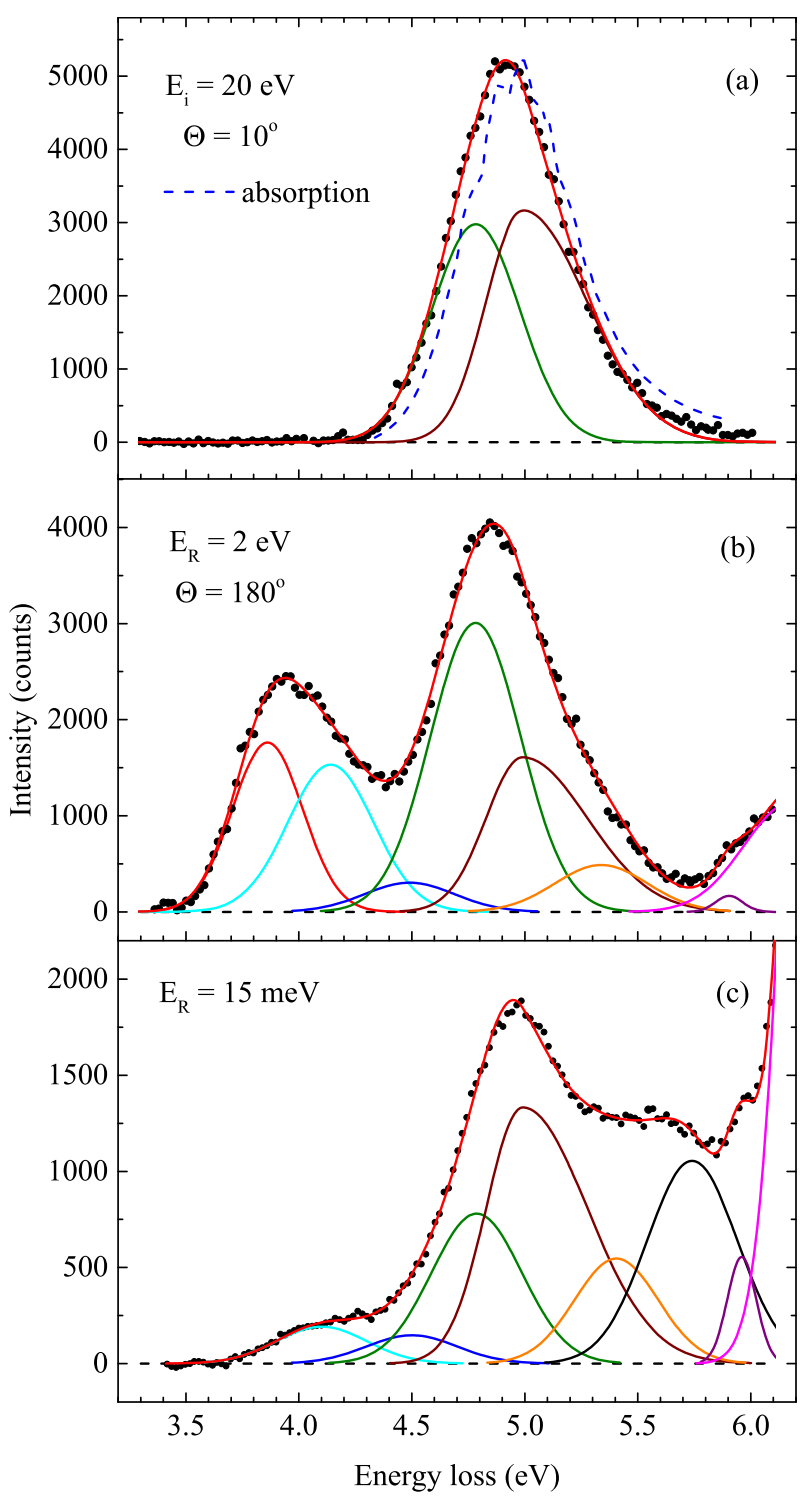

Fig. 4. Electron energy loss spectra measured in pyridine at: (a) $E_{R}=20 \mathrm{eV}$ and $\Theta=10^{\circ}$, (b) $E_{R}=2 \mathrm{eV}$ and $\Theta=$ $180^{\circ}$, (c) $E_{R}=15 \mathrm{meV}$ (threshold spectrum). Excitation bands fitted to the experimental spectra and their final fits are shown by full lines. Colour code of the bands: red $-1^{3} \mathrm{~A}_{1}$, cyan $-1^{3} \mathrm{~B}_{1}$, blue $-1^{3} \mathrm{~B}_{2}$, green $-1^{1} \mathrm{~B}_{1}$, brown $-1^{1} \mathrm{~B}_{2}$, orange $-1^{3} \mathrm{~A}_{2}, 1^{1} \mathrm{~A}_{2}$, black - Feshbach resonance. In (a) absorption measurements of Bolovinos et al. [11] are shown by blue dash line as read from their Figure 1b.

their value by analyzing the fluorescence excitation spectrum measured by Yamazaki and Baba [42]. The width of the ${ }^{1} \mathrm{~B}_{1}$ band (FWHM) obtained from the fitting procedure is $0.47 \mathrm{eV}$. The width of the ${ }^{1} \mathrm{~B}_{2}$ band, approximated by the bi-Gaussian function, was taken in the fitting to be $0.53 \mathrm{eV}$, a value deduced from the absorption spectrum of Bolovinos et al. [11]. In order to reduce the number of fitted parameters, the vertical excitation energy of the ${ }^{1} \mathrm{~B}_{2}$ state was maintained at $4.99 \mathrm{eV}$ as obtained in reference [11].

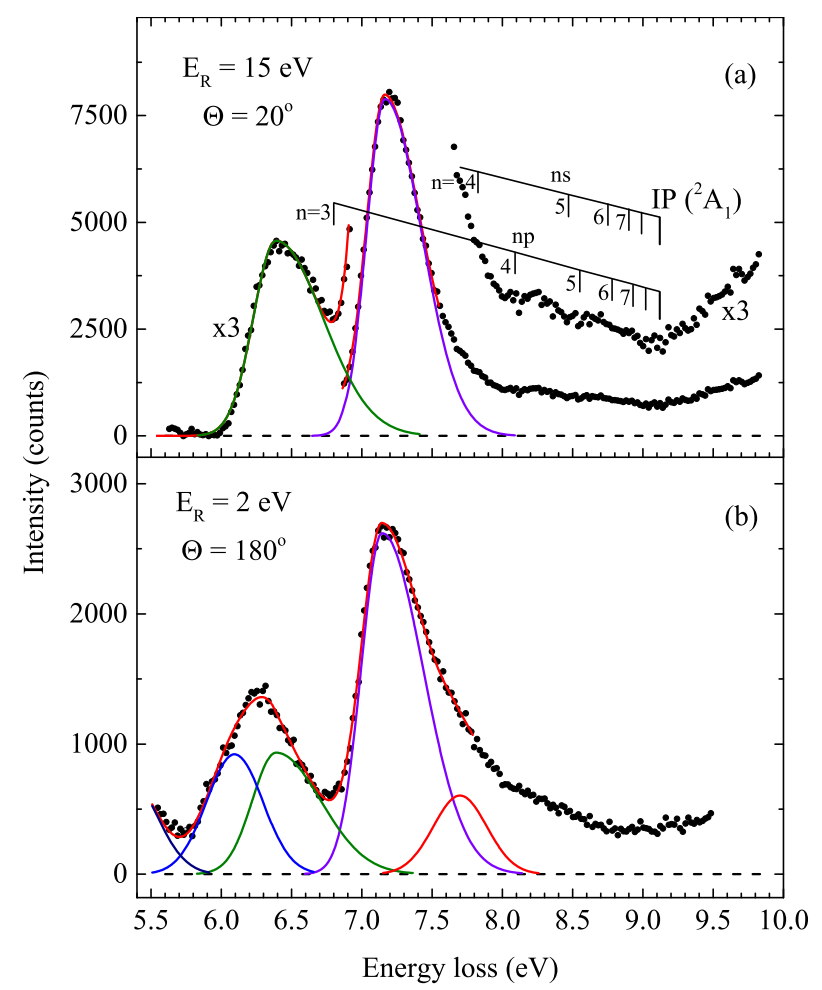

Fig. 5. Electron energy loss spectra measured in pyridine at: (a) $E_{R}=15 \mathrm{eV}$ and $\Theta=20^{\circ}$, (b) $E_{R}=2 \mathrm{eV}$ and $\Theta=180^{\circ}$. Excitation bands fitted to the experimental spectra and their final fits are shown by full lines. In (a) energies of the $n^{-1} n s$ and $n^{-1} n p$ Rydberg series observed by Bolovinos et al. [11] and first ionization potential IP are indicated by vertical bars.

The next single optical band, whose shape agrees very well with that of the corresponding $\pi \pi^{*}{ }^{1} \mathrm{~A}_{1}$ band at $6.38 \mathrm{eV}$ in the UV spectra $[11,12]$, has been recorded in the energy-loss spectra measured for $E_{i} \geqslant 20 \mathrm{eV}$ and $\Theta=10$, $20^{\circ}$ (Figs. 1 and $5 \mathrm{a}$ ). It was fitted with a single bi-Gaussian function with a maximum energy of $6.39 \mathrm{eV}$ (see Fig. 5a). The band shows weak vibrational structure with an average spacing of $100 \mathrm{meV}$. The highest intensity band in the UV spectrum occurs at $7.22 \mathrm{eV}[11,12]$ and has been attributed to the $\pi \pi^{*}{ }^{1} \mathrm{~A}_{1}$ and ${ }^{1} \mathrm{~B}_{2}$ states using a correlation with the ${ }^{1} \mathrm{E}_{2 u}$ state in benzene. The present energy-loss spectra at $E_{i} \geqslant 20 \mathrm{eV}$ contain a band with a maximum at $7.20 \mathrm{eV}$ (Figs. 1 and 5a), whose position and shape, except for the vibrational structure, closely coincide with those seen in the optical spectra. It was again successfully fitted with a bi-Gaussian function with a somewhat lower energy of $7.14 \mathrm{eV}$ (see Fig. 5a).

The first two triplet states of pyridine have been identified in the present spectra at 3.86 and $4.12 \mathrm{eV}$ (vertical excitation energies). They are assigned to $\pi \pi^{*}{ }^{3} \mathrm{~A}_{1}$ and $n \pi^{*}$ ${ }^{3} \mathrm{~B}_{1}$ symmetries, respectively, following the prediction of most computational methods that the ${ }^{3} \mathrm{~A}_{1}$ state lies more than $0.1 \mathrm{eV}$ below the ${ }^{3} \mathrm{~B}_{1}$ state [3]. Buma et al. [17] have shown that because the lowest triplet state of pyridine is nonplanar, there is a strong vibronic coupling between the ${ }^{3} \mathrm{~A}_{1}$ and ${ }^{3} \mathrm{~B}_{1}$ states. The excitation of both states is 
Table 1. Vertical excitation energies (in electron volts) of the electronic states of pyridine.

\begin{tabular}{|c|c|c|c|c|c|c|c|c|c|c|}
\hline \multirow{2}{*}{\multicolumn{2}{|c|}{ Assignment }} & \multicolumn{3}{|c|}{ Experiment } & \multicolumn{6}{|c|}{ Calculations } \\
\hline & & \multirow{2}{*}{$\frac{\text { Present work }}{3.86}$} & \multirow[t]{2}{*}{ UV [11] } & EEL [12] & $\begin{array}{c}\text { TD-DFT } \\
{[29]} \\
\end{array}$ & $\begin{array}{c}\text { CASPT2 } \\
{[3]} \\
\end{array}$ & $\begin{array}{c}\text { CASPT2 } \\
{[28]} \\
\end{array}$ & $\begin{array}{l}\text { CC3 } \\
{[27]} \\
\end{array}$ & $\begin{array}{c}\text { SAC-CI } \\
{[23]} \\
\end{array}$ & $\begin{array}{l}\text { SAC-CI } \\
{[25,26]} \\
\end{array}$ \\
\hline$\left(\pi \pi^{*}\right)$ & $1^{3} A_{1}$ & & & 4.1 & 3.91 & 4.10 & 4.05 & 4.25 & 4.06 & 4.12 \\
\hline$\left(n \pi^{*}\right)$ & $1^{3} \mathrm{~B}_{1}$ & 4.12 & & 4.1 & 4.05 & 4.37 & 4.41 & 4.50 & 4.25 & 4.46 \\
\hline$\left(\pi \pi^{*}\right)$ & $1^{3} \mathrm{~B}_{2}$ & 4.47 & & 4.84 & 4.47 & 4.61 & 4.56 & 4.86 & 4.64 & 4.79 \\
\hline$\left(n \pi^{*}\right)$ & $1^{1} \mathrm{~B}_{1}$ & 4.78 & $4.74^{\mathrm{a}}$ & & 4.76 & 5.00 & 4.91 & 5.05 & 4.59 & 4.90 \\
\hline$\left(\pi \pi^{*}\right)$ & $1^{1} \mathrm{~B}_{2}$ & 4.99 & 4.99 & & 5.47 & 4.89 & 4.84 & 5.15 & 4.85 & 5.07 \\
\hline$\left(\pi \pi^{*}\right)$ & $2^{3} \mathrm{~A}_{1}$ & & & 4.84 & & 4.77 & 4.73 & 5.05 & 4.91 & 5.08 \\
\hline$\left(n \pi^{*}\right)$ & $1^{3} A_{2}$ & 5.40 & & 5.4 & 4.93 & 5.22 & 5.10 & 5.46 & 5.28 & 5.44 \\
\hline \multirow[t]{2}{*}{$\left(n \pi^{*}\right)$} & $1^{1} \mathrm{~A}_{2}$ & 5.40 & & 5.43 & 5.07 & 5.25 & 5.17 & 5.50 & 5.11 & 5.39 \\
\hline & & 5.74 & & & & & & & & \\
\hline$\left(\pi \pi^{*}\right)$ & $2^{3} \mathrm{~B}_{2}$ & 6.09 & & & & & 6.02 & 6.40 & 6.08 & \\
\hline \multirow[t]{2}{*}{$(n 3 s)$} & $1^{1} \mathrm{~A}_{1}$ & $6.25^{\mathrm{b}}$ & $6.28^{\mathrm{c}}$ & 6.3 & & & 6.70 & & 6.17 & 6.31 \\
\hline & $2^{1} \mathrm{~A}_{1}$ & $\begin{array}{l}6.3 \\
6.39\end{array}$ & 6.38 & & 6.19 & & 6.42 & 685 & 6.26 & 6.48 \\
\hline$\left(\pi \pi^{*}\right)$ & $3^{1} \mathrm{~A}_{1}$ & $\begin{array}{l}0.09 \\
7.20\end{array}$ & $\begin{array}{l}0.00 \\
7.22\end{array}$ & 7.2 & 0.19 & & $\begin{array}{l}0.42 \\
7.23\end{array}$ & $\begin{array}{l}0.80 \\
7.70\end{array}$ & $\begin{array}{l}0.20 \\
7.18\end{array}$ & 0.48 \\
\hline \multirow[t]{3}{*}{$\left(\pi \pi^{*}\right)$} & $2^{1} \mathrm{~B}_{2}$ & 7.20 & 7.22 & 7.2 & & & 7.48 & 7.59 & 7.27 & \\
\hline & & 7.7 & & & & & & & & \\
\hline & & 8.3 & & & & & & & & \\
\hline
\end{tabular}

${ }^{\mathrm{a}}$ Estimated in reference [3]; ${ }^{\mathrm{b}}$ estimated from Feshbach resonance energy; ${ }^{\mathrm{c}}$ appearance energy from reference [44].

seen in the present energy-loss spectra for $E_{R} \leqslant 4 \mathrm{eV}$ (Fig. 4b) which emphasizes their triplet character. However, the threshold spectra $\left(E_{R}<100 \mathrm{meV}\right)$ show excitation of just the higher-lying state (Fig. 4c) indicating that the ${ }^{3} \mathrm{~B}_{1}$ state has a higher excitation cross section at threshold than the ${ }^{3} \mathrm{~A}_{1}$ state. In the spectra measured at the backward scattering $\left(\Theta=180^{\circ}\right)$, an intense energyloss peak at $4.0 \mathrm{eV}$ is seen for $E_{R}=2$ and $4 \mathrm{eV}$. Interestingly however, the peak reduces to a lower intensity shoulder for $E_{R}=3 \mathrm{eV}$. The likely explanation for the variation in the intensity and shape of the $4.0 \mathrm{eV}$ peak is excitation of both triplet states via core-excited shape resonances located at about 6 and $8 \mathrm{eV}$. It is of note, that in benzene Allan [43] has observed excitation of the triplet states to be strongly dominated by resonances in the $10 \mathrm{eV}$ range above their thresholds. The present more comprehensive energy-loss spectra support the earlier observations of a triplet peak at $4.1 \mathrm{eV}$ in the electron impact studies by Doering and Moore [20] and Walker et al. [12].

The third triplet state is deduced from the measured energy-loss spectra at $4.47 \mathrm{eV}$. Its excitation is discerned in the threshold spectra for $E_{R}=15 \mathrm{meV}$ as a rather weak shoulder (Fig. 4c) but becomes more apparent when analyzing the energy-loss region between 4.0 and $4.9 \mathrm{eV}$ (Fig. 4b). The electron intensity with respect to that of the 4.9 peak in the $\Theta=180^{\circ}$ spectra obtained for $E_{R}$ increased between 2 and $4 \mathrm{eV}$ increases showing more intense excitation of the $4.47 \mathrm{eV}$ state. The state is assigned to the ${ }^{3} \mathrm{~B}_{2}$ symmetry. The CASPT2 [3,28], SAC-CI [23] and TD-DFT [29] computational methods predict the energy of the ${ }^{3} \mathrm{~B}_{2}$ state to be slightly higher but within $0.15 \mathrm{eV}$ of the present value (see Tab. 1). The singlet-triplet splitting obtained for the $\mathrm{B}_{1}$ and $\mathrm{B}_{2}$ symmetries is 0.65 and $0.51 \mathrm{eV}$, respectively.
In the energy region $5.2-5.8 \mathrm{eV}$, the threshold spectra $\left(E_{R}\right.$ varied between 15 and $\left.100 \mathrm{meV}\right)$ show enhanced intensity of the scattered low energy electrons. These fragments in the spectra are consistently fitted with two Gaussian peaks having maxima at 5.40 and $5.74 \mathrm{eV}$ (Fig. 4c). The $5.74 \mathrm{eV}$ peak is recorded only in the threshold spectra (compare spectra in Fig. 2) and its intensity decreases fast with the increasing $E_{R}$. The intensity of the $5.40 \mathrm{eV}$ peak, on the other hand, decreases more slowly with the increasing $E_{R}$. The peak is further seen, although with low intensity, in the energy-loss spectra obtained for lower $E_{R}(\leqslant 4 \mathrm{eV})$ but not in those measured to observe the optically-allowed transitions (Fig. 4). Thus, the $5.40 \mathrm{eV}$ band is assigned to excitation of the symmetry forbidden ${ }^{3} \mathrm{~A}_{2}$ and ${ }^{1} \mathrm{~A}_{2}$ states assuming that the singlettriplet splitting, as predicted by the calculations $[3,27,28]$ (see Tab. 1), is small (below $0.1 \mathrm{eV}$ ). It may be anticipated, however, that the triplet ${ }^{3} \mathrm{~A}_{2}$ state will dominate in the $5.40 \mathrm{eV}$ band in the spectra measured for the backward scattering. The $5.74 \mathrm{eV}$ peak is attributed to a Feshbach resonance, which ejecting threshold electrons decays to an excited state. This excited state is weakly excited directly as it is not observed in the energy-loss spectra for $E_{R}>0.1 \mathrm{eV}$. The Feshbach resonance may also decay to high vibrational levels of the lower lying valence states of pyridine (e.g. the $\mathrm{A}_{2}$ states). Such resonance excitation mechanism was earlier observed in benzene by Allan [43], in the same energy range, who correlated the $e_{1 g} 3 s^{2}$ Feshbach resonance with the $3 s$ Rydberg state as a parent. In pyridine, the $n 3 s^{1} \mathrm{~A}_{1}$ Rydberg state was observed by Turner et al. [44] at $6.28 \mathrm{eV}$ applying one color $(2+1)$ and by Tsubouchi and Suzuki [45] at $6.26 \mathrm{eV}$ (value corrected using the $9.1978 \mathrm{eV}$ ionization energy) applying $\left(1+2^{\prime}\right)$ REMPI techniques. By analogy, it may be expected 
that the Rydberg $n 3 s$ state in pyridine is the parent state of the $5.74 \mathrm{eV}$ resonance, which will have a binding energy of about $0.5 \mathrm{eV}$ and may be assigned tentatively to the $n 3 s^{2}$ configuration.

The $6.4 \mathrm{eV}$ band in the $E_{\mathrm{R}}=2 \mathrm{eV}$ and $\Theta=180^{\circ}$ spectrum is reproduced by two curves, the bi-Gaussian profile of the $\pi \pi^{*}{ }^{1} \mathrm{~A}_{1}$ state and a fitted Gaussian profile with a maximum at $6.09 \mathrm{eV}$, which describes reliably the shift of the origin of the band to $5.7 \mathrm{eV}$ (Fig. 5b). The low energy slope of the band shows a weak vibrational structure, which arises at $5.9 \mathrm{eV}$. The $5.9 \mathrm{eV}$ structure is seen more clearly, as a peak, in the threshold spectra (see Figs. 3 and 4c) indicating excitation of the $6.09 \mathrm{eV}$ state also in the threshold spectra. The $6.09 \mathrm{eV}$ state is tentatively assigned to the triplet $2^{3} \mathrm{~B}_{2}$ state as it is observed for low $E_{R}$ and high $\Theta$ and is predicted in this energy range by computations $[23,27,28]$. The threshold spectra display, however, a high intensity peak at $6.35 \mathrm{eV}$ (Fig. 2), which indicates strong additional excitation at threshold of valence states of pyridine. The peak may also contain excitation of the $n 3 s$ Rydberg state.

The fitting of the $7.2 \mathrm{eV}$ band recorded in the spectra for $E_{\mathrm{R}} \leqslant 3 \mathrm{eV}$ (Fig. 5b) and also in the threshold spectra reveals that it is wider than that in the spectra of optically-allowed transitions (Fig. 5a). It demonstrates excitation of higher lying valence states of pyridine in the region around $7.7 \mathrm{eV}$. Indeed, pyridine excited triplet states of the ${ }^{3} \mathrm{~A}_{1}$ and ${ }^{3} \mathrm{~B}_{2}$ symmetries have been foreseen in the calculations in the $7.2-7.8 \mathrm{eV}$ region $[27,28]$. Moreover, the threshold spectra show excitation of further valence states at $8.3 \mathrm{eV}$ (see Fig. 2). Finally, a weak excitation of the Rydberg series in pyridine is recorded in the spectrum of Figure 5a, which also presents the energy positions of the singlet $n^{-1} n s$ and $n^{-1} n p$ series observed in absorption by Bolovinos [11].

\section{Conclusions}

The electron energy-loss spectra have been measured in pyridine at the residual electron energy varied in a wide range and at the scattering angles in the range from $10^{\circ}$ to $180^{\circ}$. The varied conditions of the electron scattering measurements enabled to differentiate between opticallyallowed and -forbidden transitions. The spectra have been analyzed using a fitting routine to resolve the excitation bands of the electronic states of pyridine. This approach permitted to observe excitation of a number of triplet states and to determine their vertical excitation energies. The energies of the first two triplet states, $\pi \pi^{*}{ }^{3} \mathrm{~A}_{1}$ and $n \pi^{*}{ }^{3} \mathrm{~B}_{1}$, are 3.86 and $4.12 \mathrm{eV}$, respectively. From comparison of determined excitation energies with results of calculations, it may be concluded that the most advanced computations provide excitation energies of the electronic states within about $0.2 \mathrm{eV}$ of the experimental values.

\section{Author contribution statement}

The authors contributed equally to the present paper.
This paper is dedicated to Prof. Michael Allan on the occasion of his retirement in recognition to his great scientific contribution to the field of electron scattering.

\section{References}

1. V.A. Lobastov, R. Srinivasan, B.M. Goodson, C.-Y. Ruan, J.S. Feenstra, A.H. Zewail, J. Phys. Chem. A 105, 11159 (2001)

2. M. Chachisvilis, A.H. Zewail, J. Phys. Chem. A 103, 7408 (1999)

3. Z.-L. Cai, J.R. Reimers, J. Phys. Chem. A 104, 8389 (2000)

4. D. Zhong, E.W.-G. Diau, T.M. Bernhardt, S. de Feyter, J.D. Roberts, A.H. Zewail, Chem. Phys. Lett. 298, 129 (1998)

5. S. Trajmar, J.K. Rice, A. Kuppermann, Adv. Chem. Phys. 18, 15 (1970)

6. M. Allan, J. Electron. Spectrosc. Rel. Phenom. 48, 219 (1989)

7. W.A. Goddard III, D.L. Huestis, D.C. Cartwright, S. Trajmar, Chem. Phys. Lett. 11, 329 (1971)

8. S.-Y. Liu, K. Alnama, J. Matsumoto, K. Nishizawa, H. Kohguchi, Y.-P. Lee, T. Suzuki, J. Phys. Chem. 115, 2953 (2011)

9. Y.R. Lee, D.W. Kang, H.L. Kim, C.H. Kwon, J. Chem. Phys. 141, 174303 (2014)

10. M. Riese, Z. Altung, J. Grotemeyer, Phys. Chem. Chem. Phys. 8, 4441 (2006)

11. A. Bolovinos, P. Tsekeris, J. Philis, E. Pantos, G. Andritsopoulos, J. Mol. Spectrosc. 103, 240 (1984)

12. I.C. Walker, M.H. Palmer, A. Hopkirk, Chem. Phys. 141, 365 (1989)

13. U. Ölsher, R. Lubart, M. Brith, Chem. Phys. 17, 237 (1976)

14. S. Japar, D.A. Ramsay, J. Chem. Phys. 58, 5832 (1973)

15. K. Sushida, M. Fujita, T. Takemura, H. Baba, J. Chem. Phys. 78, 588 (1983)

16. J.I. Selco, P.L. Holt, R.B. Weisman, J. Chem. Phys. 79, 3269 (1983)

17. W.J. Buma, E.J.J. Groenen, J. Schmidt, R. de Beer, J. Chem. Phys. 91, 6549 (1989)

18. M.N. Pisanias, L.G. Christophorou, J.G. Carter, D.L. McCorkle, J. Chem. Phys. 58, 2110 (1973)

19. E.H. Van Veen, F.L. Plantenga, Chem. Phys. Lett. 30, 28 (1975)

20. J.P. Doering, J.H. Moore Jr., J. Chem. Phys. 56, 2176 (1972)

21. Ph. Avouris, J.E. Demuth, J. Chem. Phys. 75, 4783 (1981)

22. K.K. Innes, I.G. Ross, W.R. Moomaw, J. Mol. Spectrosc. 132, 492 (1988)

23. J. Wan, M. Hada, M. Ehara, H. Nakatsuji, J. Chem. Phys. 114, 5117 (2001)

24. O. Kitao, H. Nakatsuji, J. Chem. Phys. 88, 4913 (1988)

25. D. Bousquet, R. Fukuda, P. Maitarad, D. Jacquemin, I. Ciofini, C. Adamo, M. Ehara, J. Chem. Theory. Comput. 9, 2368 (2013)

26. D. Bousquet, R. Fukuda, D. Jacquemin, I. Ciofini, C. Adamo, M. Ehara, J. Chem. Theory. Comput. 10, 3969 (2014) 
27. M. Schreiber, M.R. Siva-Junior, S.P.A. Sauer, W. Thiel, J. Chem. Phys. 128, 134110 (2008)

28. J. Lorentzon, M.P. Fülscher, B.O. Roos, Theor. Chim. Acta 92, 67 (1995)

29. R. Bauernschmitt, R. Ahlrichs, Chem. Phys. Lett. 256, 454 (1996)

30. S. Knuts, O. Vahtras, H. Ågren, J. Mol. Struct. (Teochem) 279, 249 (1993)

31. M. Nooijen, Spectrochim. Acta A 55, 539 (1999)

32. J.E. Del Bene, J.D. Watts, R.J. Bartlett, J. Chem. Phys. 106, 6051 (1997)

33. M.P. Fülscher, K. Andersson, B.O. Roos, J. Phys. Chem. 96, 9204 (1992)

34. J.C. Gibson, R.J. Gulley, J.P. Sullivan, S.J. Buckman, V. Chan, P. D. Burrow, J. Phys. B 29, 3177 (1996)

35. I. Linert, M. Zubek, J. Phys. B 39, 4087 (2006)

36. I. Linert, G.C. King, M. Zubek, J. Electr. Spectrosc. Rel. Phenom. 134, 1 (2004)

37. S. Cvejanović, F.H. Read, J. Phys. B 7, 1180 (1974)
38. M. Zubek, M. Dampc, I. Linert, T. Neumann, J. Chem. Phys. 135, 134317 (2011)

39. R.K. Nesbet, Phys. Rev. A 12, 444 (1975)

40. B.-O. Jonsson, E. Lindholm, Int. J. Mass Spectrom. Ion Phys. 3, 385 (1969)

41. I. Linert, M. Zubek, Chem. Phys. Lett. 624, 1 (2015)

42. I. Yamazaki, H. Baba, J. Chem. Phys. 66, 5826 (1977)

43. M. Allan, Helv. Chim. Acta 65, 2008 (1982)

44. R.E. Turner, V. Vaida, C.A. Molini, J.O. Berg, D.H. Parker, Chem. Phys. 28, 47 (1978)

45. M. Tsubouchi, T. Suzuki, J. Phys. Chem. A 107, 10897 (2003)

Open Access This is an open access article distributed under the terms of the Creative Commons Attribution License (http://creativecommons.org/licenses/by/4.0), which permits unrestricted use, distribution, and reproduction in any medium, provided the original work is properly cited. 